\title{
Notice historique sur les roches moutonnées, polies et striées
}

Par Jean-Pierre Portmann

Il s'agit ici de quelques références bibliographiques et citations relatives aux observations faites en Suisse, au siècle dernier, sur le modelé qu'on reconnut alors comme glaciaire.

1834: Charpentier, Jean de (1834), Notice sur la cause probable des transports de blocs erratiques de la Suisse. Actes de la Société helvétique des sciences naturelles.

1835: - Idem (Notice sur...). Annales des Mines de Paris VIII, p.219, et aussi Bibliothèque universelle de Genève IV, p.1 (1836) [traduit en allemand in Mittheilungen aus dem Gebiete der theoretischen Erdkunde, Zürich 1835, p.482].

1837: Agassiz, Louis (1837), Discours d'ouverture du 24 juillet 1837. Actes de la Société helvétique des sciences naturelles, p. V-XXXII (p. X-XV: roches polies).

Schimper, Karl (1837), Auszug aus dem Briefe des Herrn Dr. Schimper Ueber die Eiszeit... Actes de la Société helvétique des sciences naturelles, p. 38-51 (p.45-49: polis glaciaires).

1838: [Agassiz, Louis]: «En 1838, vers la fin d'août, c'est une première randonnée dans les Alpes, principalement à la recherche de roches moutonnées, de polis glaciaires, de moraines anciennes.» Portmann, J.-P., Louis Agassiz (1807-1873) et l'étude des glaciers. Mémoires de la Société helvétique des sciences naturelles $L X X X I X, 1973$, p. 121.

Une inscription: «1838 L. Agassiz Eisschliff» - $30 \times 5 \mathrm{~cm}-$ le long de l'ancien chemin du Grimsel (Helle Platten, 667090/161650/1510 m) rappelle cette excursion: J. of Glaciology (1961-1963), p.38. In: Portmann, J.-P. (1973), op. cit., p.132, note $24^{1}$.

Cf. Desor, Ed., Excursions et séjours dans les glaciers et les hautes régions des Alpes de M. Agassiz et de ses compagnons de voyage. Neuchâtel, Paris 1844 , p. 16 («Stries rectilignes»); p. 41 («Fines stries, cannelures et sillons»); p. 42 («Stries»).

1839: [Agassiz, Louis]: «Du Riffelberg, c'est la découverte sublime du vaste panorama glaciaire. A proximité du glacier, Agassiz montre des roches polies et striées à Bernard Studer... qui, incrédule, n'admet pas encore la 
théorie glaciaire. Par contre, le guide, Joseph Brantschen de Zermatt, fait remarquer tout naturellement que seul le glacier pouvait user de cette façon. Studer est convaincu ${ }^{2}$ ' On ne peut plus douter, c'est chose démontrée', déclare-t-il en promettant de publier lui-même ce qu'il venait de voir.» - Portmann, J.-P. (1973), Louis Agassiz (1807-1873) et l'étude des glaciers, p. 122 .

Cf. Desor, Ed., Excursions et séjours dans les glaciers et les hautes régions des Alpes de M. Agassiz et de ses compagnons de voyage, 1844, p. 74-75. Cf. Studer, B., Bulletin Soc. Géol. de France (1) IX (1837-1838), p.407, 443-450.

1840: Agassiz, Louis (1840), Etudes sur les glaciers. Neuchâtel et Soleure, 1840.

- Ch. XIV: Des actions des glaciers sur leur fond

«Les glaciers polissent la roche sur laquelle ils reposent» (p.188) cf. pl. 17, pl.18, fig. 2 .

«Différence entre le poli produit par la glace et celui qui est produit par l'eau» (p.190).

«On sait, dit-il [de Charpentier] que les glaciers frottent, usent et polissent les roches avec lesquelles ils sont en contact»... «cependant j'ignore que cette observation ait été faite par qui que ce soit avant lui. Il paraît que M. de Saussure n'en avait pas connaissance; il n'a du moins pas eu l'idée de rattacher cette action des glaciers aux roches polies du Grand St. Bernard qu'il attribue à l'action de l'eau» (p.190).

«Stries que l'on remarque sur les roches polies» (p.193).

[en note, p. 195: allusion aux observations à ce sujet de M. Sefstroem, en Suède.]

- Ch. XVII: Les roches polies

Allusion à celles d'Oberwald signalées par Guyot (p.251) - pl.9, pl.14, pl. 16.

Desor, Ed. (1840), Voyage au Mt-Rose et au Cervin. Bibliothèque universelle de Genève, mai 1840 (p.33).

Mousson, Alb. (1840), Geologische Skizze der Umgebung von Baden im Kanton Aargau (p.90).

1841: Charpentier, Jean de (1841), Essai sur les glaciers et sur le terrain erratique du Bassin du Rhône. Lausanne 1841.

«Nous voulons parler de ces marques de frottement sur lesquelles nous avions déjà appelé l'attention des géologues dans notre petit mémoire de 1834 [p.15]. Ces marques consistent dans l'usure et l'aspect lisse de la surface des rochers»... (p.166, §56). 
«...M. Agassiz en a donné la description» [Discours d'ouverture, 1837, p.XI] (p. 167).

«...marques de frottement»...«on reconnaît quelquefois sur ces derniers des stries tellement fines »...

«Ces stries, parallèles entre elles, et non à la pente de la montagne, mais plutôt à celle du sol de la vallée, semblent être rayées au moyen d'un fin burin »... (p. 168).

1844: Agassiz, Louis (1844), Notice sur les glaciers (p.1-14), in: Desor, Ed. (1844), Excursions et séjours dans les glaciers et les hautes régions des Alpes de M. Agassiz et de ses compagnons de voyage. Neuchâtel, Paris 1844 (p. 15-638). Agassiz: «... et gravent sur les surfaces polies une multitude de stries rectilignes plus ou moins fines et sensiblement parallèles entre elles»... (p.10).

\section{Notes}

${ }^{1}$ Voir aussi: Aerni, Klaus: Gemmi-Loetschen-Grimsel. Beiträge zur bernischen Paßgeschichte. Jahrb. Geogr. Gesell. Bern 51 (1973-74), Fig.8, p.40. - Il existe un article spécial sur cette inscription dans la revue Die Alpen. Elle est représentée aussi dans Gesnerus 31 (1974), entre p. 8 et 9.

${ }^{2}$ Des échantillons de ces serpentines polies et striées furent prélevés; «l'un de ces morceaux a été figuré sur la planche 18 de l'atlas de M. Agassiz, qui représente des roches polies de différentes localités » (Desor, 1844, p. 74, note 1).

Prof. Dr. Jean-Pierre Portmann

Institut de Géographie

Université de Neuchâtel

10, Coq-d'Inde

CH-2000 Neuchâtel 